\title{
An Association of Mucinous Adenocarcinoma With Chronic Peri-Anal Fistula: A Brief Review of Pathophysiology of Rare Tumor
}

\author{
Muhammad Tahir ${ }^{1}$, Jawaria Rahman ${ }^{2}$, Tayyaba Zubair ${ }^{3}$, Abdul Basit ${ }^{3}$ \\ 1. Pathology, Case Western Reserve University School of Medicine, Cleveland, USA 2. Pathology, City of Hope, \\ Comprehensive Cancer Center, Monrovia, USA 3. Internal Medicine, Coney Island Hospital, Brooklyn, USA
}

Corresponding author: Muhammad Tahir, tahir_786kb@yahoo.com

\begin{abstract}
An anal fistula is a condition that has been discussed by various authors without coming to a consensus. The fistula of the anus is a passage that leads from the rectum to the anus. A peri-anal adenocarcinoma is an abnormal growth of cells in the anal rectal area. Establishing the relationship between the two, a lot of information was obtained from the existing literature which has used to come up with solutions to the objective of the study. The review was conducted systematically and included observational retrospective, case studies, and case series to demonstrate the data of valuable research. The articles were searched in PubMed, MEDLINE, and Google scholar using the keywords "chronic perianal fistula", "anal carcinoma", "mucinous adenocarcinoma", and "perianal mucinous adenocarcinoma”. Among 50 journal articles, we chose 33 studies describing the clinical sign and symptoms, pathophysiology, etiology, and association between mucinous anal adenocarcinoma and chronic peri-anal fistula. After quality assessment, eight case studies and series were selected, in which seven of them showed the origin of mucinous anal adenocarcinoma from chronic peri-anal fistula. The existence of a long history of fistula-in-ano and the exclusion of any additional carcinoma in the body necessitates the analysis of mucinous adenocarcinoma arising from benign fistula. Likewise, the presence of fistula ought to typically precede that of carcinoma by 10 years, and this is one of the criteria for diagnosis. However, more investigation should be conducted to gain full information about the connection between these two entities.
\end{abstract}

Received 06/08/2020 Review began 06/19/2020 Review ended 06/22/2020 Published 06/28/2020

(c) Copyright 2020 Tahir et al. This is an open access article distributed under the terms of the Creative Commons Attribution License CC-BY 4.0., which permits unrestricted use, distribution, and reproduction in any medium, provided the original author and source are credited.
Categories: Internal Medicine, Pathology, Oncology

Keywords: chronic peri-anal fistula, anal carcinoma, mucinous carcinoma, peri-anal mucinous adenocarcinoma, mucinous adenocarcinoma

\section{Introduction And Background}

Peri-anal fistulas can be found frequently in the clinical setting, but the malignant transformation of these fistulas is not common. The development of anal mucinous adenocarcinoma (MA) from chronic peri-anal fistula is rare, and only a few such cases have been reported in the past [1]. Anal fistulas usually develop from an anal abscess, and the background of the fistula is regularly very typical. The patient with anal fistulas, along with abscess, generally has signs and symptoms of peri-anal swelling, pain with defecation, fever, lethargy, purulent discharge, and rectal discomfort [2]. Overall, 37\% of patients develop a fistula from an anal abscess. The occurrence of anal fistula is higher in females than in males with $50 \%$ and $31 \%$, respectively [3].

Cancer of the peri-anal fistula is an erratic disease. It is predominantly associated with chronic anal fistula. The MA accounts for $2-3 \%$ of all anorectal malignancies. In most cases, prolonged duration inflammation is the possible reason for fistula-related carcinomas [4]. Diagnosis is frequently not made on time because the disease appears very slowly with fewer symptoms. The neoplasm tends to develop into large masses before they can be identified [5].

MA is a diverse clinical and pathological entity within the range of colorectal neoplasms and reports nearly $10-15 \%$ of cases. Causes related to MA growth and progress have not been entirely known, but clinical observational evidence can guide us to the best understanding of its etiology [6]. To satisfy the definition of MA as highlighted by the World Health Organization, more than $50 \%$ of the tumor should comprise extracellular mucin [7]. Chronic inflammation presumably plays a significant role in carcinogenesis. Colorectal carcinomas (CRCs) occur primarily in areas affected by inflammation, but the mechanism of carcinogenesis is not yet totally understood [8-10]. As indicated by some studies, the peri-anal MA is linked to chronic anal fistula. Howbeit, that is not always the case; very limited research has been conducted to investigate the connection between peri-anal fistula and MA. Plenty of other studies will need to be performed to have a complete picture of this relationship.

There are worldwide differences in the incidence of mucinous carcinomas (MCs), with low rates in Asian countries and increased rates in the western world. Furthermore, MC is more often diagnosed in patients suffering from inflammatory bowel diseases or Lynch syndrome, and a high rate has been seen in patients 
with radiotherapy-induced CRCs. These outcomes are indicative of various oncogenic origin [6]. The purpose of this review is to perform an analysis of the emergence of MA from chronic anal fistula and understand the pathophysiology of this rare malignancy.

\section{Review}

\section{Method}

We performed this study systematically using the Preferred Reporting Items for Systematic Reviews and Meta-Analyses (PRISMA) [11]. To search for the information, we used the various resources but mainly selected PubMed for gathering the majority of the data. Resources such as MEDLINE, WHO website, PubMed Central, WebMD, and Google Scholar were also searched. The keywords used for searching included chronic peri-anal fistula, anal carcinoma, MC, adenocarcinoma (AD), and peri-anal MA. We concentrated on the adult population from all over the globe without prejudice between gender, race, nationality, and ethnicity. Nevertheless, several publications demonstrating a correlation among various age groups have also been added. Among all relevant articles, a quality assessment checked was performed using A MeaSurement Tool to Assess Systematic Reviews (AMSTAR) checklist, and multiple publications were omitted [12]. All data was collected scientifically and ethically.

\section{Result}

We retrieved 2,119 studies from PubMed and 16,900 from Google scholar studies using the search term "chronic peri-anal fistula" and "mucinous anal adenocarcinoma". The combined use of the search term "relationship between chronic peri-anal fistula and mucinous anal adenocarcinoma” provided us with 13,303 PubMed and 18,500 Google Scholar research papers. A total of 50 articles related to our subject were picked from these findings. After implementing the exclusion/inclusion criteria and removing the repetition, we ultimately got 33 research papers that were considered for review. The majority of the related articles that were selected were case studies and series, which discussed the clinical sign and symptoms, pathophysiology and etiology, and the relationship between chronic peri-anal fistula and mucinous anal $\mathrm{AD}$. After careful consideration, eight case studies and series were chosen, in which seven of them demonstrated the association between chronic peri-anal fistula and mucinous anal AD.

\section{Discussion}

An anal fistula (often called fistula-in-ano) is most of the time a consequence of a past or current anal abscess. This occurs in up to half of patients with abscesses. Typical anatomy consists of small glands directly inside the anus. The fistula is the channel that builds beneath the skin and links the blocked infected glands to an abscess. A fistula can exist without an abscess and may connect to the skin of the buttocks close to the anal opening. Fistulas have categorized through their association with the parts of the anal sphincter complex (the muscle that controls stool to pass). They are as follows: superficial fistula, transsphincteric, extrasphincteric, suprasphincteric, and intersphincteric, as shown in Figure 1. The most common one is the intersphincteric, and the least common is extrasphincteric. These classifications are necessary for assisting the physicians in making their treatment choices [13]. 


\section{Cureus}

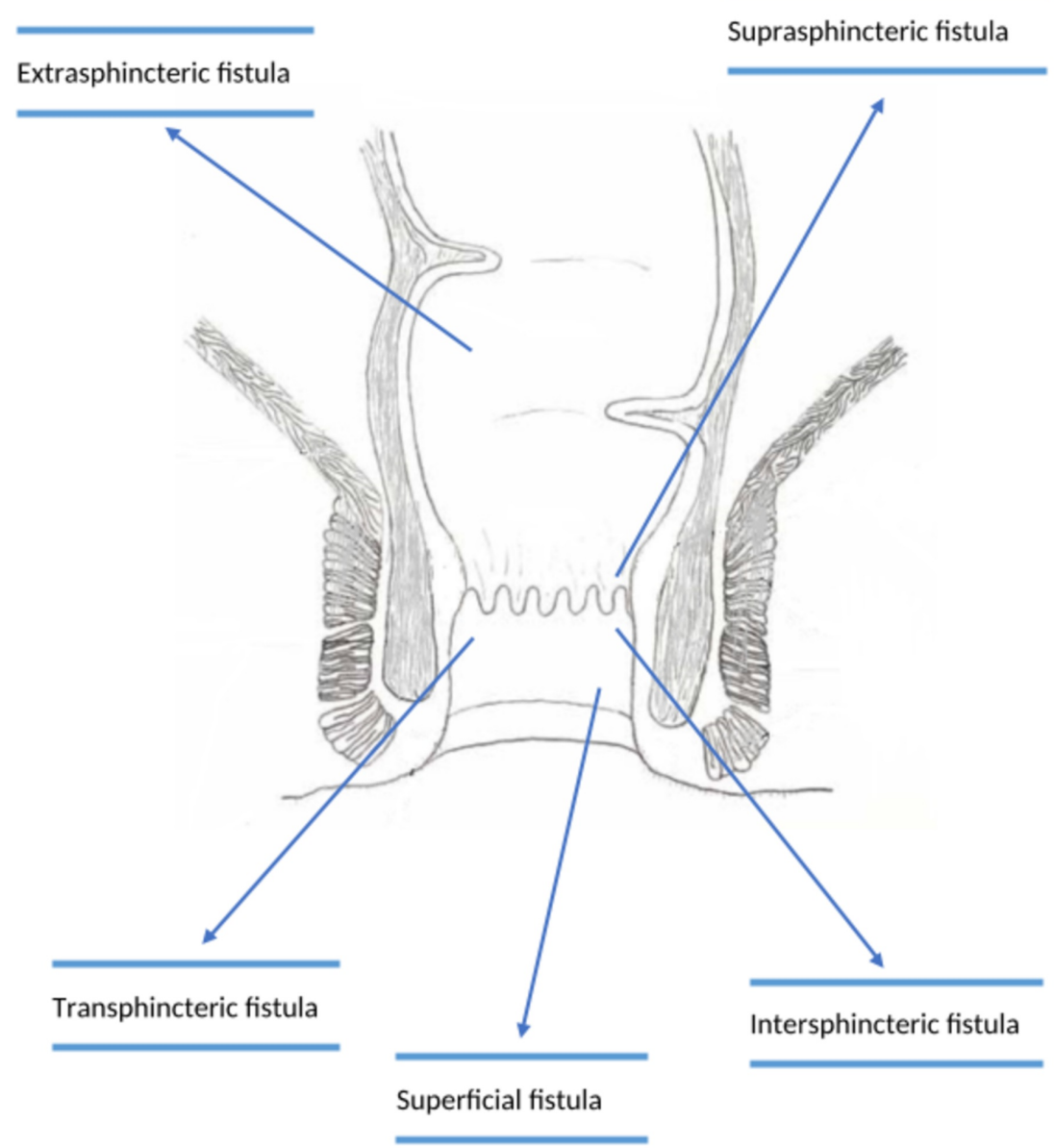

FIGURE 1: Classification of peri-anal fistula

The emergence of MA from peri-anal fistula is still perplexing. The area around anus consists of both squamous and columnar epithelium, and therefore carcinoma can be squamous cell carcinoma or AD [14].

The anal canal acts as an anatomical transition area between the peri-anal squamous mucosa and the columnar epithelium. The underlying pathophysiology that leads to malignant transformation is still unknown, but the chronic inflammation with frequent epithelial regeneration has considered instrumental [15].

MA is a subcategory of colorectal cancer, accounting for almost $10 \%$ of all colorectal cancer (CRC), as shown in Figure 2, and roughly 2-3\% peri-anal cancers are MAs, as shown in Figure 3 [16,17]. 


\section{Cureus}

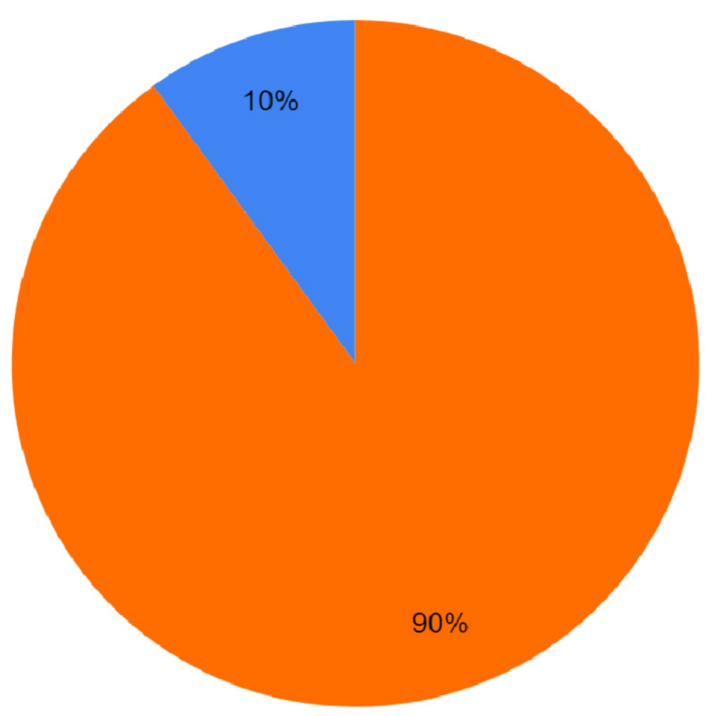

Colorectal Carcinomas (CRC)

Mucinous Adenocarcinomas (MA)

FIGURE 2: Ratio of mucinous adenocarcinoma relative to all colorectal cancers

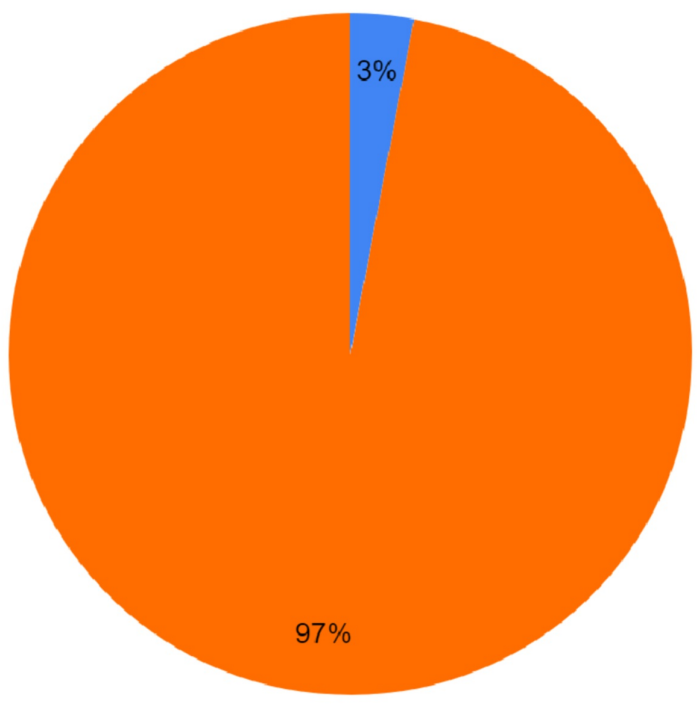

Mucinous Adenocarcinoma

Peri-anal Cancers

FIGURE 3: Ratio of mucinous adenocarcinoma relative to peri-anal carcinomas

This sort of cancer comprises malignant cells that form an enormous quantity of extracellular mucin, and the histopathological identification needs to have more than $50 \%$ mucinous element of the tumor mass [7]. The prognostic findings of MA patients stayed questionable. Few investigators have put forward that patients with colorectal MA have a poorer prognosis than those with $\mathrm{AD}$, though others have indicated no discrepancy [17-22].

The most common causes of MC are inflammatory bowel disease and Lynch syndrome, and a higher rate is seen in patients with radiotherapy-induced CRCs [6]. A handful of cases have indicated the origin of MA from chronic peri-anal fistula.

Further details revealed the different techniques to diagnose the MA, which includes endoanal ultrasonography (EAUS), which is the most efficient one and shows the specific attributes of MA arising from chronic peri-anal fistula. Sonography-guided biopsy under anesthesia is quite beneficial for the definitive diagnosis of the tumor. In this manner, regular evaluation with EAUS ought to be suggested for patients with long-standing peri-anal fistula, particularly those with dynamic clinical manifestations. Once the 
malignancy is suspected, vigorous sonography-guided biopsy under anesthesia should be carried out, which may empower the early findings, treatment, and favorable long-term benefits [23].

MCs of the colon and rectum grow and metastasize faster than non-MCs. It is believed that the pathological and clinical aspects resulted in decrease curability, consequent relapse, and an adverse prognosis. For that reason, surgeons recognized that more proactive surgical treatment, including a wide range of lymph node dissection and resection of adjacent organs that are probably involved, must be delivered from time to time to better the postoperative prognosis of the patients with colorectal MC [24].

The pathogenesis has not yet been entirely explained. Traube et al. described the theory of constant mucosal regeneration within the fistula, which can lead to dysplastic changes that may result in developing AD [25]. Symonds et al. suggested that the neoplasm categorized as MC is made up of glands secreting pools of mucus deep inside the infiltrating part of the tumor [26]. Pihl et al. stressed that MC should be limited to neoplasms in which mucin prevails [27]. Moreover, Wu et al. and Connelly et al. mentioned that a tumor in which the mucinous portion is at least $60 \%$ of the neoplasm should be considered as an MC [28,29]. The biologic importance of MC of the colon and rectum continued to be contentious [30,31]. Overall, the prognosis of the patients diagnosed with colorectal MC is worse [23].

However, many studies have been conducted to clarify the pathogenesis of MA and its origin from chronic peri-anal fistula. Yet, we still have a gap in establishing the precise diagnosis and need more clinical-based evidence to prove the association between two. A study conducted by Santos et al. conducted a study on patients with constant peri-anal fistulas who gradually grew two peri-anal ulcerated sores close to the outer orifices of the fistulas, ultimately reaching out as a pararectal tumor. The histopathological biopsy confirmed the diagnosis of MA [32]. Another study was conducted by Haliloğlu et al. to study the results of magnetic resonance imaging (MRI) of MA arising from peri-anal fistula, and based on the study, MRI is the choice of imaging technique for the diagnosis of MA arising from peri-anal fistula. The study also reported that a fistula tract biopsy of the two patients with a history of chronic peri-anal fistula showed MA [15]. In another study, Prasad et al. reported the origin of MA in a patient with chronic anal fistula, which was confirmed by transrectal ultrasound-guided biopsy and consecutive histopathological examinations. The study summed up three criteria needed for the diagnosis of MA: (1) the presence of fistula ought to usually precede that of carcinoma by 10 years, (2) any tumorous association of the rectum or anal canal should only be a secondary extension of the primary mass involving the fistula, and (3) internal opening of the fistula within the rectum or anal canal should not be included, as shown in Figure 4 [1].

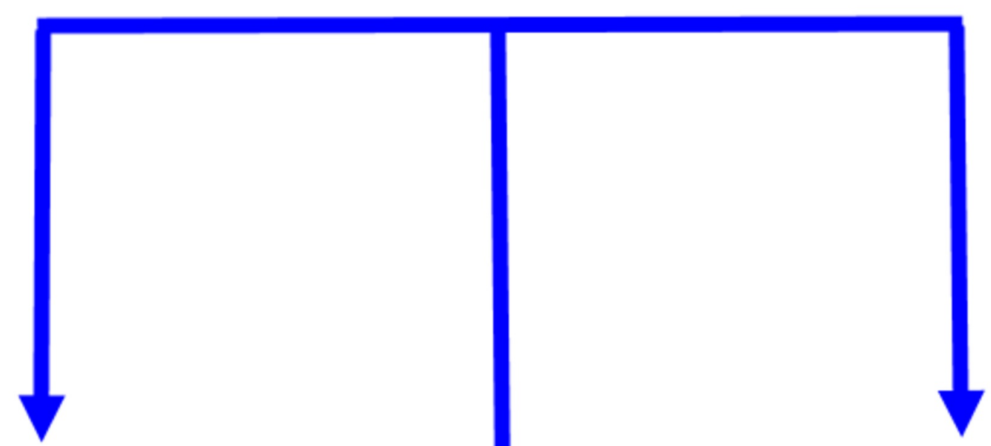

The presence of fistula should usually antedate that of carcinoma by ten years
Internal opening of the fistula within the rectum or anal canal should not be involved

\author{
Any tumorous \\ involvement of the \\ rectum or anal canal \\ should only be a \\ secondary extension of \\ the primary mass \\ involving the fistula
}

FIGURE 4: Three-point criteria presented by Prasad et al. emphasizing an association of mucinous adenocarcinoma with chronic peri-anal fistula 


\section{Cureus}

Toyonaga et al. showed the significance of EAUS of MA arising from chronic peri-anal fistula. The study demonstrated a typical pathology of MA with multiloculated complex echoic mass along isoechoic solid components communicating with a trans-sphincteric fistula, as shown by a sonography-guided biopsy under anesthesia [23]. Another study was conducted by Papaconstantinou et al. which reported the root of the MA within a chronic peri-anal fistula in Crohn's disease. The study proved that the constant mucosal regeneration inside a fistula in Crohn's disease tends to be a dominant pathogenetic mechanism. The mucosal biopsies collected from the lesions and the underlying fistulous tracts showed granulomatous tissue infiltrated by mucus-producing AD [33]. The study by Díaz-Vico et al. involved three patients and demonstrated that all three patients with chronic peri-anal fistula had developed MA over time. In the study, they emphasized that histopathological diagnosis remains the gold standard method of diagnosis. The presence of extracellular mucinous lakes encompassed by well-differentiated dilated tortuous glands, nerves, and vessels confirmed the diagnosis of MA [34]. One more study conducted by Bo-Lin Yang et al. based on retrospective case reviews identified three patients with chronic peri-anal fistula who were subsequently found to developed peri-anal MA on biopsy [35]. Table 1 summarizes all the aforementioned studies. 


\section{Cureus}

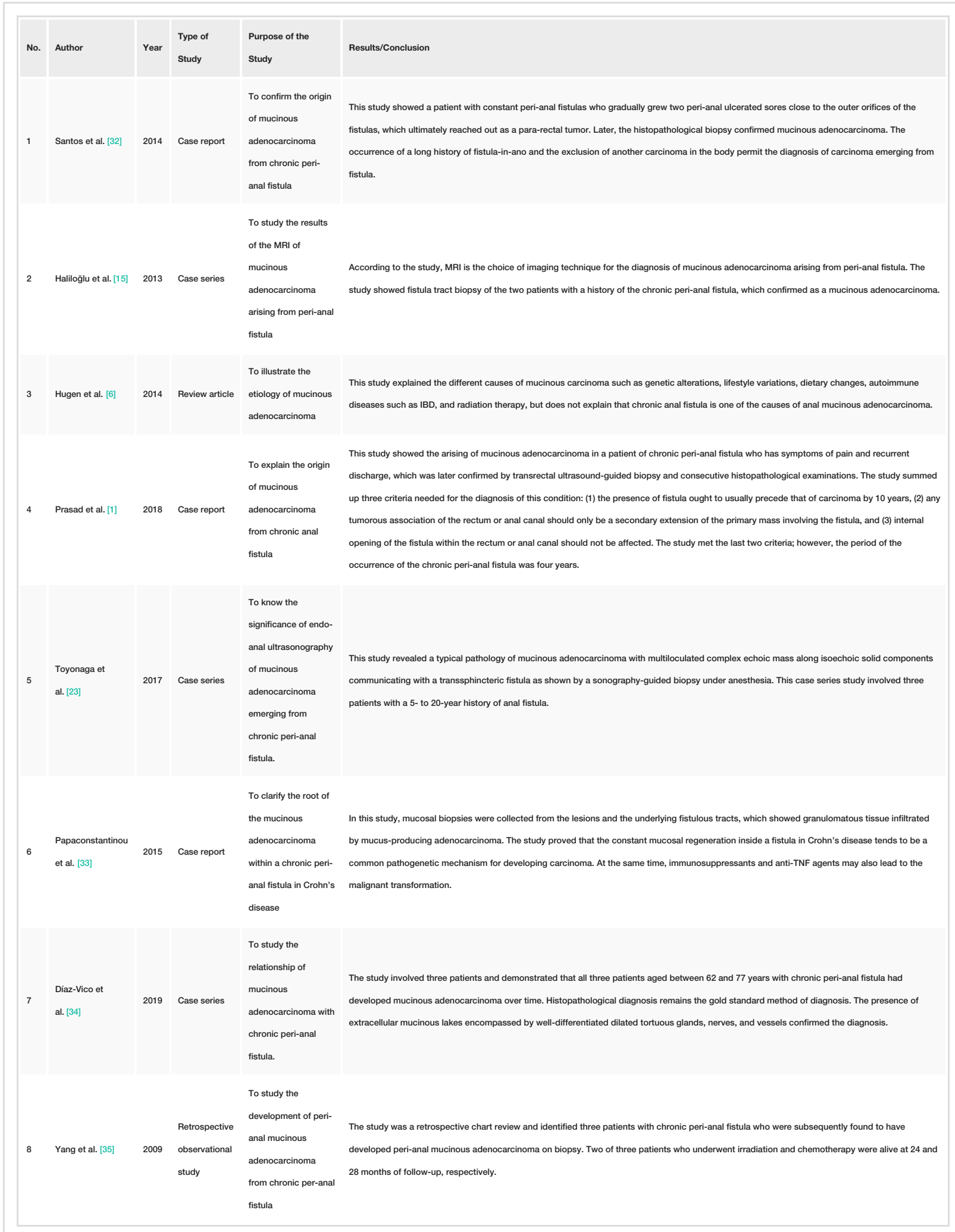

TABLE 1: An association of mucinous adenocarcinoma with chronic peri-anal fistula in relevant studies

IBD, inflammatory bowel disease; TNF, tumor necrosis factor

Based on the studies reviewed, MA arising from long-standing peri-anal fistulas is uncommon. However, the occurrence of a long history of fistula-in-ano and the exclusion of another carcinoma in the body allows the diagnosis of MA emerging in a benign fistula. Also, the presence of fistula should usually antedate that of 
carcinoma by 10 years, and this is one of the pieces of evidence found in studies. On gross analysis, the tumor appears tan whitish to pinkish with cut-surface, might be germinal scar or necrosis, usually welldefined, and could be capsulated, smooth, cystic, or solid with even distribution with or without modularity. Furthermore, chronic mucosal regeneration plays a vital role in the pathogenesis of MA. The confirmatory diagnosis of mucinous $\mathrm{AD}$ includes the histopathological findings of the presence of extracellular mucin encircled by dilated tortuous glands.

\section{Limitations}

The research has various limitations that could affect the accuracy of the results. Foremost, the number of people with the condition is very minimal, thus making it impossible to get enough data for analysis. Most of the investigation was based on a small sample size in their report. Another limitation could be that the research was conducted based only on the literature. Thus, the reliability of the information or data was dependent on already existing research studies. Another limitation is that the condition is very hard to diagnose until the symptoms usually manifest themselves during the last stages, and the duration of time to follow the results of an association between the two entities makes the researcher bore or quit from the study. Therefore, the delayed analysis could compromise the accuracy of the results.

\section{Conclusions}

MA arising from chronic peri-anal fistula is an uncommon occurrence. Still, it is visible that the two conditions have an association with each other, where the chronic disease causes the other, though not all the times. Understanding this relationship may be challenging, especially the diagnosis, as the signs manifest themselves at the later stages. The condition can be controlled if it is diagnosed early enough. A high level of clinical suspicion is required for early diagnosis, which builds the extension for therapeutic medical and surgical procedures. Imaging is very helpful in identifying the growing malignancy and staging the disease, thereby directing early and effective treatment. The histopathological assessment is prudent to neglect the chance of missing an underlying malignancy. Establishing the relationship between the two conditions will assist in decreasing the tumor burden for the hospital, patient, and government beforehand, and will also help focus on the patients to improve their living standards and handle and endure their chronic illness in comparison with the suffering of complications of malignancy, which will deteriorate their life physically, mentally, and monetarily.

\section{Additional Information \\ Disclosures}

Conflicts of interest: In compliance with the ICMJE uniform disclosure form, all authors declare the following: Payment/services info: All authors have declared that no financial support was received from any organization for the submitted work. Financial relationships: All authors have declared that they have no financial relationships at present or within the previous three years with any organizations that might have an interest in the submitted work. Other relationships: All authors have declared that there are no other relationships or activities that could appear to have influenced the submitted work.

\section{Acknowledgements}

I would like to thank Jawaria Rahman for her endless efforts and help she offered to me for this article. It was a pleasure working with her. She is an awesome team player and a very kind human being.

\section{References}

1. Prasad SN, Razik A, Siddiqui F, Lal H: Mucinous adenocarcinoma arising from chronic perianal fistula mimicking horseshoe abscess. BMJ Case Rep. 2018, 2018:bcr2017223063. 10.1136/bcr-2017-223063

2. Abcarian H: Clinical assessment of anal fistulas. Anal Fistula. Abcarian H (ed): Springer, New York, NY; 2014. 27-29. 10.1007/978-1-4614-9014-2_5

3. Hämäläinen KP, Sainio AP: Incidence of fistulas after drainage of acute anorectal abscesses . Dis Colon Rectum. 1998, 41:1357-1362. 10.1007/BF02237048

4. Lee T, Yong E, Ding NS: Radiological outcomes in perianal fistulizing Crohn's disease: a systematic review and meta-analysis. JGH Open. 2019, 4:340-344. 10.1002/jgh3.12295

5. Zhu ZB: Primary perianal mucinous adenocarcinoma: a case report and literature review . World Chinese J Digest. 2016, 24:653-656. 10.11569/wcjd.v24.i4.653

6. Hugen N, van Beek JJ, de Wilt JH, Nagtegaal ID: Insight into mucinous colorectal carcinoma: clues from etiology. Ann Surg Oncol. 2014, 21:2963-2970. 10.1245/s10434-014-3706

7. World Health Organization: World Health Organization Classification of Tumours: Pathology and Genetics of Tumours of the Digestive System. Hamilton SR, Aaltonen LA (ed): IARC Press, Lyon, France; 2010.

8. Choi PM, Zelig MP: Similarity of colorectal cancer in Crohn's disease and ulcerative colitis: implications for carcinogenesis and prevention. Gut. 1994, 35:950-954. 10.1136/gut.35.7.950

9. Svrcek M, Cosnes J, Beaugerie L, Parc R, Bennis M, Tiret E, Fléjou JF: Colorectal neoplasia in Crohn's colitis: a retrospective comparative study with ulcerative colitis. Histopathology. 2007, 50:574-583. 10.1111/j.13652559.2007.02663.X

10. Hussain SP, Amstad P, Raja K, et al.: Increased p53 mutation load in noncancerous colon tissue from ulcerative colitis: a cancer-prone chronic inflammatory disease. Cancer Res. 2000, 60:3333-3337. 
11. Moher D, Liberati A, Tetzlaff J, Altman DG: The PRISMA Group (2009). Preferred Reporting Items for Systematic Reviews and Meta-Analyses: the PRISMA statement. PLoS Med. 2009, 6:e1000097. 10.1371/journal.pmed.1000097

12. Shea BJ, Reeves BC, Wells G, et al.: AMSTAR 2: a critical appraisal tool for systematic reviews that include randomised or non-randomised studies of healthcare interventions, or both. BMJ. 2017, 358:j4008. 10.1136/bmj.j4008

13. Abscess and Fistula. Accessed: May 24, 2020: https://fascrs.org/patients/diseases-and-conditions/az/abscess-and-fistula.

14. Hu X, Li YQ, Li QG, Ma YL, Peng JJ, Cai S: Mucinous adenocarcinomas histotype can also be a high-risk factor for stage II colorectal cancer patients. Cell Physiol Biochem. 2018, 47:630-640. 10.1159/000490018

15. Haliloğlu, Nuray, Esra Özkavukçu, and Ayşe Erden: MRI findings of mucinous adenocarcinoma arising from perianal fistula: report of two cases. Gazi Med J. 2013, 24:87-89. 10.12996/gmj.2013.25

16. Consorti F, Lorenzotti A, Midiri G, Di Paola M: Prognostic significance of mucinous carcinoma of colon and rectum: a prospective case-control study. J Surg Oncol. 2000, 73:70-74. 10.1002/(sici)10969098(200002)73:2<70::aid-jso3>3.0.c0;2-j

17. Green JB, Timmcke AE, Mitchell WT, Hicks TC, Gathright JB Jr, Ray JE: Mucinous carcinoma--just another colon cancer?. Dis Colon Rectum. 1993, 36:49-54. 10.1007/BF02050301

18. Kanemitsu Y, Kato T, Hirai T, et al.: Survival after curative resection for mucinous adenocarcinoma of the colorectum. Dis Colon Rectum. 2003, 46:160-167. 10.1007/s10350-004-6518-0

19. Purdie CA, Piris J: Histopathological grade, mucinous differentiation and DNA ploidy in relation to prognosis in colorectal carcinoma. Histopathology. 2000, 36:121-126.

20. Nitsche U, Friess H, Agha A, et al.: Prognosis of mucinous and signet-ring cell colorectal cancer in a population-based cohort. J Cancer Res Clin Oncol. 2016, 142:2357-2366. 10.1007/s00432-016-2224-2

21. Catalano V, Loupakis F, Graziano F, et al.: Prognosis of mucinous histology for patients with radically resected stage II and III colon cancer. Ann Oncol. 2012, 23:135-141. 10.1093/annonc/mdr062

22. Yarbro JW, Page DL, Fielding LP, Partridge EE, Murphy GP: American Joint Committee on Cancer prognostic factors consensus conference. Cancer. 1999, 86:2436-2446.

23. Toyonaga T, Mibu R, Matsuda H, et al.: Endoanal ultrasonography of mucinous adenocarcinoma arising from chronic fistula-in-ano: three case reports. J Anus Rectum Colon. 2018, 1:100-105. 10.23922/jarc.2017011

24. Nozoe T, Anai H, Nasu S, Sugimachi K: Clinicopathological characteristics of mucinous carcinoma of the colon and rectum. J Surg Oncol. 2000, 75:103-107. 10.1002/1096-9098(200010)75:2<103::aid-jso6>3.0.co;2-c

25. Traube J, Simpson S, Riddell RH, Levin B, Kirsner JB: Crohn's disease and adenocarcinoma of the bowel . Digest Dis Sci. 1980, 25:939-944. 10.1007/BF01308045

26. Symonds DA, Vickery AL: Mucinous carcinoma of the colon and rectum. Cancer. 1976, 37:1891-1900. 10.1002/1097-0142(197604)37:4<1891::aid-cncr2820370439>3.0.co;2-z

27. Pihl E, Nairn RC, Hughes ESR, Cuthbertson AM, Rollo AJ: Mucinous colorectal carcinoma: immunopathology and prognosis. Pathology. 1980, 12:439-447. 10.3109/00313028009077107

28. Wu CS, Tung S, Chen P, Kuo YC: Clinicopathological study of colorectal mucinous carcinoma in Taiwan: a multivariate study. J Gastroenterol Hepatol. 1996, 11:77-81. 10.1111/j.1440-1746.1996.tb00014.x

29. Connelly JH, Robey-Cafferty SS, Clearry KR: Mucinous carcinomas of the colon and rectum. An Analysis of 62 Stage B and C Lesions. Arch Pathol Lab Med. 1991, 115:1022-1025.

30. Rankin FW, Chumley CL: Colloid carcinoma of the colon and rectum. Arch Surg. 1929, 18:129-139. 10.1001/archsurg.1929.04420010131006

31. Kim YS, Ho SB: Intestinal goblet cells and mucins in health and disease: recent insights and progress . Curr Gastroenterol Rep. 2010, 12:319-330. 10.1007/s11894-010-0131-2

32. Santos MD, Nogueira C, Lopes C: Mucinous adenocarcinoma arising in chronic perianal fistula: good results with neoadjuvant chemoradiotherapy followed by surgery. Case Rep Surg. 2014, 2014:386150. $10.1155 / 2014 / 386150$

33. Papaconstantinou I, Mantzos DS, Kondi-Pafiti A, Koutroubakis IE: Anal adenocarcinoma complicating chronic Crohn’s disease. Int J Surg Case Rep. 2015, 10:201-203. 10.1016/j.ijscr.2015.04.013

34. Díaz-Vico T, Fernández-Martínez D, García-Gutiérrez C, et al.: Mucinous adenocarcinoma arising from chronic perianal fistula-a multidisciplinary approach. J Gastrointest Oncol. 2019, 10:589-596. 10.21037/jgo.2019.01.11

35. Yang BL, Shao WJ, Sun GD, Chen YQ, Huang JC: Perianal mucinous adenocarcinoma arising from chronic anorectal fistulae: a review from single institution. Int J Colorectal Dis. 2009, 24:1001-1006. 10.1007/s00384-009-0657-7 